\title{
Assessing Spanish-Speaking University Students' Info-Competencies with iSkills, SAILS, and an In-House Instrument: Challenges and Benefits
}

\author{
Jesus Lau \\ Juan D. Machin-Mastromatteo \\ Alberto Gárate \\ A. Cecilia Tagliapietra-Ovies
}

This article was originally published as:

Lau, J., Machin-Mastromatteo, J.D., Gárate, A., and Tagliapietra-Ovies, C. (2016). Assessing Spanish-speaking university students' info-competencies with iSkills, SAILS, and an in-house instrument: Challenges and benefits. Communications in Computer and Information Science, 676, 327-336. https://doi.org/10.1007/978-3-319-52162-6 32

\begin{abstract}
In its ongoing assessment of information literacy competencies (ILC), CETYS Universidad first developed an in-house instrument and then applied the standardized tests, SAILS and iSkills. This paper analyzes the design of these instruments as well as data gathered from their implementation. This comparison aids in finding similarities in the ILC they measure, describe how they measure them, and study their differences. The objectives of this analysis are to describe the evolution of the Institution's ILC assessment over the past four years while providing the basis for making an evidence-based decision about improving the in-house instrument and pushing to develop a Spanish-language multi-institutional tool, and/or the continued use of international instruments.
\end{abstract}

Keywords: Information Literacy · Competencies · Assessment

\section{Introduction}

The assessment of information literacy competencies (ILC) is a must for any university that has the strategic goal of helping students learn how to evaluate the quality and reliability of information. The need for measuring students' ILC is related to other needs that libraries and educational institutions have, such as increased accountability, assessment and accreditation. Measuring ILC involves determining the degree to which students are information literate and asking if their information literacy attainment influences their success while finding out the library's role in their information proficiency and asserting whether the resources dedicated to the library and to information literacy (IL) programs are sufficient [1, 2]. Evaluation usually comes at the end of librarians' and educators' IL concerns [6] and scattered experiences have not been published. There are many assessment instruments available and reported in the literature, primarily from English-speaking countries. Most of them are tailored to institutional needs; iSkills, the Standardized Assessment of Information Literacy Skills (SAILS), and the Madison Research Essential Skills Test (MREST) tests stand out. The use of such tools designed in the United States and commercially available is one option 
for non-English speaking universities, such as Mexican ones, with few professional library staff or resources to develop their own instruments. Available lists of instruments [3,4] do not include any instrument in Spanish, a language spoken in more than 20 countries. Assessing ILC becomes a challenge in Spanish-speaking universities because there are no standardized or formalized tools available for review and use in the Ibero- American region. Although the development of IL instruments has been a concern in Ibero-American universities, few have attempted to create testing tools because of the complexity of this task [5]. Using these international tests is a challenge given the language, culture and information resource differences; but locally developed tests present outcome shortcomings [7].

CETYS Universidad is a three-campus educational institution, located in three cities in Baja California, Mexico (Mexicali, Tijuana, Ensenada). It offers high-school, Bachelors, and Masters degrees. One of its strategic goals is to be an information culture- oriented organization and, thus, it has been assessing students' ILC. First, CETYS developed and used an in-house instrument. Then, it administered a pilot version of SAILS and, finally, it implemented iSkills. This paper presents CETYS' experience with these IL instruments and an analysis of their characteristics and their results using the in-house instrument [8], SAILS [1] and iSkills [9]. These objective instruments allow evaluating whether students are, indeed, developing ILC and they complement other measurement materials [10] such as rubrics and evidences while evaluating the benefits of other institutional IL initiatives that have aimed to develop an information culture.

\section{CETYS Experience with IL Instruments}

CETYS formally started to conduct Institutional Assessment in 2008 as an answer to the Western Association of Schools and Colleges' (WASC) recommendations about the need for providing evidence of students' performance. Hence, the Institutional Learning Results (RAI) were established to provide evidence of the progress of Bachelors and Masters students' academic performance. In 2014, faculty adapted RAI to integrate the Distinctive Elements of CETYS Education (EDEC) as well as the Core Competencies that WASC, as an accreditation agency, required. RAl's first block of competencies include: (a) Spanish oral and written communication; (b) information culture (also an EDEC); (c) critical thinking; and (d) quantitative reasoning. This block is related to information management and communication skills, hence the relevance of using objecttive instruments for assessing ILC, especially for measuring information culture, which is both a Core Competence and EDEC. Such instruments are appropriate complements to other learning evidences such as essays or research reports and the rubrics used to evaluate them. They allow measuring students' level of competence regarding ILC by providing useful data on strengths and improvement opportunities. These instruments are useful for institutional assessment; in determining the impact of certain institutional milestones, such as the new Information Management course that is mandatory for all first semester students); and the reference librarians' (RL) activities. The CETYS Library System also supports ILC. The RL were hired in 2013 to work on the Information Culture Development Program (ICD) which includes actions catering to students and 
faculty such as workshops on information and research resources, tutorials and promotional materials, and libraryacademia joint activities $[10,11]$. The CETYS experience with IL instruments has had three distinct moments. In 2012, the Center for Academic Development and Improvement (CDMA), together with faculty and library staff, designed an in-house instrument for measuring first semester undergraduate and graduate students' proficiency in ILC with the desire of conducting a longitudinal study during the following years. The second initiative consisted of applying SAILS by the end of 2013 using a pilot version for the first time in a Spanish-speaking nation. The third action, which took place by the end of 2015, involved implementing iSkills, arguably the most dominant test among American universities. The following subsections describe the design and characteristics of these three instruments.

\subsection{Development of an In-House Instrument}

The CDMA, faculty, and library staff developed this instrument with the objective of identifying the ILC of students from Bachelors and Masters programs. They designed two similar questionnaires, one for first year Bachelors students and the other for first year Masters students. The instruments differed only in their demographic questions. The instrument design was guided by three basic documents:

- The Information Literacy Competency Standards for Higher Education, which state that the information literate student: "(i) determines the nature and extent of the information needed; (ii) accesses needed information effectively and efficiently; (iii) evaluates information and its sources critically and incorporates selected information into his or her knowledge base and value system; (iv) individually or as a member of a group, uses information effectively to accomplish a specific purpose; and $(\mathrm{v})$ understands many of the economic, legal, and social issues surrounding the use of information and accesses and uses information ethically and legally" [12].

- The Mexican Guidelines 'Development of Information Skills in Mexico's Higher Education Institutions', divided into eight skills that ask students to "understand information, identify need, locate and search, retrieve, evaluate, use, communicate, and ethically use information" [13].

- IFLA's Guidelines on Information Literacy for Lifelong Learning [14], which groups ILC into three main categories: access, use and evaluation.

This instrument includes demographic questions that allow profiling the surveyed students by campus, gender, age range, academic program, and in which institution they have studied before their enrollment at CETYS. Then, it includes five sections, each dedicated to one of the Association of College \& Research Libraries (ACRL) standards. It has eleven questions: five are multiple-choice questions and one is a matching question. Students have to order different lists of elements in the remaining five questions. The sections and questions cover the following content:

- Identification of the information need: students have to select cases where they know they need information, then they must order a list of information sources according to how important they are for use in assignments. 
- Access and retrieval of required information: given a research topic, students must select the keywords they would use in a search engine; then, they must identify the action they take when they do not find a book in the library; and, finally, they must select from a list the four most important elements they follow in selecting a website.

- Evaluation of information sources: students have to match a column of research topics with rows of information sources, according to which source is most appropriate for each topic; then, given a research topic, they have to select the least biased source from a list; and, finally, order the importance of actions to continue searching after an initial Google or database search has yielded no useful results.

- Applying and using information appropriately: they have to order the parts of an academic essay and discard the irrelevant options.

- Ethics and legal use of information: the two remaining questions involve arranging data from a book and an academic article to construct their references, according to the Publication Manual of the American Psychological Association (APA) [8].

\subsection{SAILS Pilot}

Librarians at Kent State University started Project SAILS to measure students' ILC [1]. It is a 90-min. questionnaire of 45 multiple-choice questions with a bank of 140 questions. It is based on two ACRL documents: Information Literacy Competency Standards for Higher Education and Objectives for Information Literacy Instruction: A Model Statement for Academic Librarians [1]. Thus, SAILS covers ACRL's performance indicators, outcomes, and objectives. The elements measured are explicitly documented on the results provided to institutions using it. SAILS' evaluation scale ranges from 0 to 1000 and uses the measurement model of item response theory and the one-parameter Rasch model. The calculation of scores involves item difficulty and student performance and can be used in building a benchmark from all the institutions' data. Then, "student responses to the items on the test are then used to determine the difficulty level of each item. Once that determination is made, student responses are analyzed to determine an average score for each group" [1, p. 2]. Hence, SAILS provides scores for groups of students and not individual scores. To make the most of SAILS, institutions should assess large samples to ensure score accuracy, conduct statistical research to support data analysis, and ensure that sampling methods are rigorous [15]. SAILS recommends that samples must be of at least 50 students and the ideal is 200 [1]. Although SAILS is built from the five ACRL standards, the fourth standard is not used, as they claim that part of it is covered by outcomes and objectives from other standards or contain some elements that are not adequate for multiple-choice questions. The competencies assessed by SAILS are grouped under seven skill sets: (a) Developing a Research Strategy (with 32 questions); (b) Selecting Finding Tools (18); (c) Searching (26); (d) Using Finding Tool Features (14); (e) Retrieving Sources (15); (f) Evaluating Sources (21); and (g) Documenting Sources (14) [1]. They also provide how questions are distributed according to ACRL standards: Standard 1 (39), Standard 2 (74), Standard 3 (21),

Standard 4 (not used), and Standard 5 (6) [1]. 


\subsection{Implementing iSkills}

iSkills is a commercial standardized test offered by the Educational Testing Service (ETS), a US-based company probably best known for the TOEFL test. iSkills is promoted as an Information and Communication Technologies (ICT) literacy test. Although presented as a way to measure digital skills, the skills measured are still closely related to ILC. iSkills contains scenario-based tasks and its scoring scale ranges from 0 to 500; students achieving at least 130 points are awarded a certificate of achievement. ETS has established three competency levels according to the score obtained: Developing (130250), Foundational (260-340), and Advanced (350-500). Although it evaluates ICT literacy skills areas, ETS provides an equivalence between iSkills Assessment Performance Indicators and the ACRL standards and performance indicators [16]. The test has different sections with items that provide students with a series of tasks they must perform; the ICT literacy skills assessed by iSkills are: Define (5 tasks), Access (7), Evaluate (8), Manage (5), Integrate (7), Create (9), and Communicate (7) [17]. ETS offers data from individual and group results.

\section{Results from the Instruments' Implementations}

Analyzing the results from each instrument allowed us to know the level of detail and accuracy of data that can be gathered about ILC and information behavior. The challenge in our analysis lied in having applied the three instruments at various times and to diverse groups that corresponded to different generations of students. If we had applied these three instruments at the same time to a large population of students we would have randomly divided the population into three smaller groups in order to administer a different instrument to each group. However, presenting a summary of the results with the three tests allowed us to point out the similarities, differences, challenges, and opportunities of each instrument as well as think about which test could be the best option in a given situation. Moreover, it informed the pedagogic and administrative strategies that can be used to facilitate and foster ILC development in students.

\subsection{In-House Instrument}

We gathered results through the in-house instrument to a sample of 365 first year Bachelors students and 104 first year Masters students. A group of faculty administered the survey through SurveyMonkey by the end of 2012 at the three campuses. Results showed that Bachelors students knew how to search for information in the library and on the Web, they searched information when they did not know about the topic, they had doubts, or they lacked information. However, first year Masters students searched for information when they did not know about the topic, when they wanted to learn something new, or if they had doubts. The Bachelors students indicated that the three most important information sources they used for their course assignments were: e-books, printed books and journals, and academic databases. Conversely, Masters students' most important sources were: classmates, their own printed books and journals, and an expert on the topic; the printed and digital resources available in the libraries were not among 
their top sources. Both groups of students know which keywords to use in search engines and, when they do not find the book they are searching for, they turned to the Web, asked librarians, or searched for a similar book. Both groups chose a website based on its contents, the organization or person responsible for the site, and the date of update. However, their critical ability for evaluating information was not optimal: both groups considered the Web as the first resource to locate information. Only $32 \%$ of Bachelors students and just half of master students identified the least biased information source among the options presented. Moreover, the majority of Masters students did not consider library resources as important sources for their assignments. When they did not find information in their first Google or database search, both groups stated that they would stop searching, but Bachelors students claimed they could try using Boolean operators, while Masters students looked for the support of an expert. Most students from each group could identify the order of the parts of an academic essay. Bachelors students partially knew about APA format: they could order the data from the book to construct its reference but they could not do it for the journal article. However, the majority of Masters students could not order the data from either the book or the journal article in order to construct the APA reference [8].

\subsection{SAILS Pilot}

In 2013, SAILS conducted a beta test in order to validate the instrument for using it internationally and, in 2014, they conducted a second round. In this pilot, more than 1.200 students from 80 academic institutions located in five countries allowed them to conform the benchmark. These countries were Bulgaria, Canada, China, Egypt, Mexico, Qatar, South Africa, and the USA. CETYS was the only Mexican and Latin American institution to participate in the first round of the beta test. It was administered in CETYS during December 2013 with a sample of 300 students from which 241 returned were valid tests. Of those 241, 144 were in Mexicali and 97 were in Tijuana. In order to make sure that students taking the test had a good proficiency in English. the RL and the coordinators and teachers of the College English and the course Advanced Communication in English administered SAILS., SAILS allowed the faculty and librarians clearly distinguish students' weaknesses and strengths regarding the assessed competencies. The staff compared the local results for each competence with the benchmarks of similar institutions offering the same maximum academic degrees and all institutions. Hence, if students' scores were equivalent, near, or above the values of the benchmark of similar institutions, it was assumed they had a good level of proficiency in that area. On the contrary, performances considerably below the benchmark, meant areas of weaknesses.

According to the SAILS Skill Sets, students performed 'about the same as the institution-type benchmark' in the following competencies: 'Using Finding Tool Features' (best) and 'Documenting Sources'. However, they performed 'worse than the institution-type benchmark' in: 'Developing a Research Strategy'; 'Selecting Finding Tools'; 'Searching'; 'Retrieving Sources'; and 'Evaluating Sources' [1]. It is relevant to high- light that SAILS report also included this assessment according to the ACRL Standards. According to these standards, students performed 'about the same as the institution-type benchmark' in Standard 5 (see Sect. 2.1). However, they performed 'worse than the 
institution-type benchmark' in Standards 1, 2, and 3 (see Sect. 2.1). An advantage of SAILS is that it allowed adding demographic questions so questions about campus, academic program and semester were added. These allowed staff to profile subgroups within the sample and compare their performances. Thus, students who performed better were from engineering programs, followed by those in social sciences and humanities programs (Law and Psychology); and the group with the lowest performance was from business and administration programs. A common strength was the 'Using Finding Tool Features' skill, while the common weakness was 'Evaluating Sources'. Tijuana scored slightly better than Mexicali. Mexicali's best score was on 'Using Finding Tool Features', while Tijuana's was 'Retrieving Sources' [1]. Compared to similar institutions, and especially given a generally higher score for the 'All Institutions' benchmark, CETYS students' results might be largely improved if some strategies to better support ILC are developed. In addition to these results, the RL could report that they could detect that evaluation of sources was indeed a weakness in CETYS students; and that a large proportion of students taking the SAILS test did not participate in ICD's activities, which by this time were active for only about six months.

\section{3. iSkills}

Staff at the three campuses for Bachelors students administered the standardized test iSkills. This test was applied for the first time in November 2015 for establishing performance parameters regarding information use and management. Results allowed staff to draw students' competency profiles and determine strategies for improvement. iSkills was implemented with a sample of 554 Bachelors students from all semesters (first, third, fifth, and seventh) from all academic programs. The ETS provided results for the 503 students finished the test. A group of $13.72 \%$ of the students who took the iSkills also took the in-house instrument three years earlier. These students were in their first semester in 2012 and were, thus, in their seventh semester when they completed the iSkills test. Faculty and librarians observed that these students exhibited a certain level of improvement. However, one limitation in the data reports from ETS was that it could not take advantage of the demographic questions since it was not possible to see details of the results per ICT literacy skill for individual students; it is only possible to see the final scores. This made it difficult to compare sub-groups' performances in iSkills with results from the other two instruments. However, individual results facilitated by ETS allowed for the comparison of the total scores of students according to their profile (program, campus, and semester). Sixteen students achieved the highest scores (350500), eleven were from the first semester and from Mexicali who were already enrolled in the Information Management course while four were from the seventh semester and one was in the fifth. Aggregate results offered by ETS established the performance of the sample of students who took the test based on 503 finished and valid tests and, similar to SAILS, it compared the sample's performance with that of a reference group of 3,115 students from all years. However, no information was provided regarding which types of students or institutions formed this reference group. CETYS students performed about the same in the Evaluate (highest skill) and Communicate skills. They performed slightly below the reference group in Create and Access (lowest skill) and their performance 
was considerably lower for Define, Manage, and Integrate (lowest skill) [9]. The highest scored campus was Mexicali, followed by Ensenada, and then Tijuana. Regarding score by academic program, the tendency emerging from SAILS results repeated with iSkills, meaning that students from engineering scored the best (average score of 414), followed by social sciences and humanities programs (395); and business and administration (366) [9]. Hence, the average scores for all programs were within the advanced level of competency. In general, all students scored the best in the Evaluate skill, which was surprising considering that it was the lowest aspect scored in SAILS. However, this was probably due to different strategies, namely: a higher institutional emphasis in information culture; teachers instructing students to use reputable sources; the continued work of the RL; and the new Information Management course.

\section{Conclusion}

Assessing students' ILC allowed us to determining the influence of information literacy on their success (and thus retention), helped document institutional results and, thus, allowed us to compare student ILC with those of students at other institutions. It also allowed us to develop strategies for improvement, justify investments, and demonstrate variations in scores that may be caused by different actions such as more IL workshops with students, the availability of tutorials and

flyers, and curricular changes that involved embedding IL in the official programs. The results of the tests allowed us to measure variables that demonstrated students' competencies, learning outcomes, and the benefits of libraries' initiatives as well as those from the academia [2]. In 2010, before assessing ILC, the strategy progressively implemented by teachers during the past six years was to use research papers and essays in order to improve students' learning and performance, both in written communication and information use. In 2012, the application of the in-house instrument drew attention to the importance of raising awareness and improve students' and teachers' usage of the printed and digital information resources that the library acquired. This attention grew progressively over time. For example, the total number of downloads from the digital library seems to be a good number to measure usage of library information resources, but when we looked at the number of students and teachers who actively used these digital information resources, this turned out to be a low percentage of the total population. Moreover, the in-house instrument also pointed toward the need for students to improve their critical ability for evaluating information sources and resources. The arrival of the RL in the Institution in 2013 resulted in an explosion in the offering of courses, workshops, training, and promotional materials through ICD. Moreover, with the application of SAILS, we observed that these ICD experiences may have helped students perform well in the test. But the RL also detected some of the challenges in the results. Our suggestions were to include ACRL's indicators, outcomes, and objectives within ICD activities, further emphasizing the evaluation of sources and search strategies. In 2015, iSkills results were comparable with those from SAILS regarding the programs with highest scores and showed an improvement regarding the competence of evaluation. However, since our students still demonstrate some ICT literacy skills below that of the reference group used by ETS, there is room 
for improvement. Arguments for the continued use of the in-house instrument include that it is a local instrument adapted to our students' profiles and CETYS institutional needs. It is a Spanish-language instrument and as an assessment method it represents a stage of maturity in the development of an institutional information culture. One of the challenges is that we may not yet be on the same level of assessment as other institutions; perhaps some ILC are not being developed at CETYS. In using a local instrument, we ourselves have set the bar for achievement. As a result, we might have been too kind to our students. Given the level of the international standards, it would be difficult to compare our results with those from other institutions using SAILS or iSkills. Regarding the design of our instrument, we designed it by competencies, but its results are not directly expressed in competencies. Due to the instrument's brevity there are few questions or items per competency. This design did not allow us to assess different elements, facets or outcomes within an ILC. This instrument is an acceptable diagnostic for new students, but it is neither adequate for measuring students' advancement toward developing their ILC nor did it enable us to compare its results with other institutions. If we decide to continue using it, it must be reviewed considering these criticisms and the experiences with the other instruments. It would need to be made considerably more exhaustive in order to diagnose different facets of ILC in more detail. This is noteworthy when looking at the other instruments: while the in-house test has only eleven items for the five ACRL skills, SAILS has 45 (from a bank of 140) for the same five skills, and iSkills has 48 tasks for seven ICT literacy skills. The advantages of using SAILS and iSkills are that they are validated and trustworthy assessment instruments used at an international level. It is useful to have evidences of their implementations in order to compare results with those from other institutions with which we can have or set agreements. This is also important for accreditation purposes. We observed limitations in the SAILS instrument during the pilot. For example, SAILS does not provide individual scores, hence limiting the analyses on these particular results. iSkills does offer individual results and by cohort (the complete sample) but the individual results only include total scores, not the individual scores per ICT literacy skill, which are only offered for the whole cohort. This also hinders the possibility of performing more detailed analyses of the data, such as analyzing the performances per ICT literacy skill of students from certain academic program or semester. A final consideration regarding the feasibility of implementing iSkills is that ETS announced in 2016 that was discontinuing this test, indicating specific dates regarding the deadlines to acquire and use it. This represents an area of opportunity for SAILS and other competitors. Furthermore, it would be important to analyze, study and transfer to other instruments and IL assessment tools what we consider is one of iSkills' main strengths in its design over other instruments: its scenario-based tasks. In an Ibero-American experience with both standardized tests, the language barrier is a concern, because it may interfere students' performance in English-language tests. Even though a student might have English proficiency, it is still a second-language and its use for academic matters may not be so developed and thus the student's interpretation on what the test is requiring from them can be altered by their English level. The complicated part of this limitation is to know if the language is affecting results or is it really the student who does not have the competency. For example, the best score in iSkills (450) is from a third semester student with the 
maximum level in English College. As there are not many scores that high, does it mean that the rest of the students do not have the competency or is it that they do not have enough English proficiency in order to do well in these kinds of tests?

\section{References}

1. Project SAILS: Results of the SAILS for CETYS Universidad (2013)

2. Schilling, K., Applegate, R.: Best methods for evaluating educational impact: a comparison of the efficacy of commonly used measures of library instruction. J. Med. Libr. Assoc. 100(4), 258-269 (2012)

3. Mueller, J.: Assessments of information literacy (2014). http://jfmueller.faculty.noctrl.edu/ infolitassessments.htm

4. Blevens, C.: Catching up with information literacy assessment: resource for program evaluation. Coll. Res. Libr. News 73(4), 202-206 (2012)

5. O'Connor, L., Radcliff, C., Gedeon, J.: Assessing Information literacy skills: developing a standardized instrument for institutional and longitudinal measurement. In: Thompson, H. (ed.) ACRL 10th National Conference, pp. 163-174. ACRL, Chicago (2015)

6. González, C.: Apuntes Relevantes sobre la Evaluación en la Alfabetización Informacional. Librínsula: La Isla de los Libros 331 (2014). http://eprints.rclis.org/23580/

7. Mery, Y., Newby, J., Peng, K.: Assessing the reliability and validity of locally developed information literacy test items. Ref. Serv. Rev. 39(1), 98-122 (2011)

8. Tagliapietra-Ovies, A.C.: Resultados Medición del Aprendizaje Institucional RAl 2 y 3 en 2012 (2013)

9. Educational Testing Service: Institutional Skill Area Report (2015)

10. Machin-Mastromatteo, J.D.: Two years of information culture development for supporting higher education: initiatives, teacher's perceptions and future actions. Commun. Comput. Inf. Sci. 552, 517-526 (2015)

11. Machin-Mastromatteo, J.D., Beltran, O., Lau, J.: Piloting a holistic information culture program: the experience of cetys universidad system of libraries. Commun. Comput. Inf. Sci. 492, 350-360 (2014)

12. Association of College \& Research Libraries: Information literacy competency standards for higher education (2000). www.ala.org/acrl/standards/informationliteracycompetency

13. Aguilar, V., Almanza, J., Cortés, J., Endean, R., García, J., Lau, J., Ríos, H.: Declaratoria: Desarrollo de Habilidades Informativas en Instituciones de Educación Superior de México. UACJ, Cd. Juarez (1997)

14. Lau, J.: Guidelines on information literacy for lifelong learning (2007). www.ifla.org/ publications/guidelines-oninformation-literacy-for-lifelong-learning

15. Lym, B., Grossman, H., Yannotta, L., Talih, M.: Assessing the assessment: how institutions administered, interpreted, and used SAILS. Ref. Serv. Rev. 38(1), 168-186 (2010)

16. Educational Testing Service: ETS Higher Education iSkills Assessment Fit with ACRL Standards (2008). www.ets.org/Media/Tests/ICT_Literacy/pdf/acrl_standards.pdf

17. Educational Testing Service: The iSkills assessment (2016). www.ets.org/iskills/about 